\title{
Dermatite Associada à Incontinência e o Uso Não Padronizado de Fraldas Geriátricas: Revisão Sistemática
}

\author{
Dermatitis Associated with Incontinence and the \\ Not-Standard Use of Geriatric Diaper: Systematic Review
}

\author{
Dermatitis Asociada con Incontinencia y Uso No \\ Estándar de Pañales Geriátricas: Revisión Sistemática
}

Luise de Almeida Ferreira Alves', Rosimere Ferreira Santana', Aluane dos Santos Cardozo',
Thaís Muniz de Souza', Carleara Ferreira da Rosa Silva ${ }^{1}$

\begin{abstract}
RESUMO
O uso de fraldas por idosos hospitalizados pode ser considerado um dos responsáveis pelo surgimento de agravos dermatológicos e exacerbação dos episódios de incontinência urinária. A ausência de padronização quanto ao seu uso e indicação contribui para a presença dos eventos adversos. Trata-se de um estudo de revisão sistemática da literatura cujo objetivo foi analisar o uso da fralda geriátrica ao que concerne a técnica, os produtos e os eventos adversos e a partir disso formular um protocolo de intervenção para uso de fraldas geriátricas. Para o direcionamento desta revisão sistemática foram seguidos os critérios do Preferred Reporting Items for Systematic Reviews and Meta-Analyses (PRISMA Statement). Para busca optou-se pelas bases de dados CINAHL, LILACS, EMBASE e MEDLINE e obteve-se amostra final de 33 artigos nas línguas portuguesa, inglesa e espanhola, sem delimitação de tempo para busca e com exclusão daqueles que abordassem sobre fraldas infantis. As pesquisas destacaram as características relacionadas à matéria prima e funcionalidades da fralda, assim como as técnicas de uso e as lacunas sobre a padronização desse processo nas intervenções de enfermagem. Do mesmo modo, os estudos demonstraram que os acometimentos cutâneos e de incontinência urinária possuem relação com a qualidade dos produtos utilizados e com a frequência das trocas.
\end{abstract}

DESCRITORES: Fraldas para adultos. Cuidados de enfermagem. Enfermagem geriátrica.

\begin{abstract}
The use of diapers for hospitalized elderly can be considered one of those responsible for the appearance of dermatological diseases and exacerbation episodes of urinary incontinence. The lack of standardization regarding the use and display contributes to the presence of adverse events. This is a systematic review study of the literature that analyzes the use of geriatric diaper as regards the technical, products and adverse events and from there formulate an intervention protocol for use of adult diapers. To the direction of this systematic review were followed the criteria of the Preferred Reporting Items for Systematic Reviews and Meta-Analyses (PRISMA Statement). For the search it was decided to use the CINAHL, LILACS, MEDLINE and EMBASE databases and it was obtained final sample of 33 articles in Portuguese, Spanish and English, without delimitation of time to search and excluding those that focused on children's diapers. The research highlighted the characteristics related to raw materials and functionality of the diaper as well as the use of techniques and gaps on the standardization of this process in nursing interventions. Similarly, studies have shown that skin affections and urinary incontinence are related to the quality of the products used and the frequency of exchanges.
\end{abstract}

DESCRIPTORS: Diapers, adult. Nursing care. Geriatric nursing. 


\section{RESUMEN}

El uso de pañales para adultos mayores hospitalizados puede ser considerado como uno de los responsables de la aparición de enfermedades dermatológicas y episodios de exacerbación de incontinencia urinaria. La falta de normalización con respecto al uso e indicación contribuye para la presencia de eventos adversos. Se trata de un estudio de revisión sistemática de la literatura que cuyo objetivo fue analizar el uso de pañales geriátricos concerniente a técnica, los productos y los eventos adversos y a partir de eso formular un protocolo de intervención para el uso de pañales geriátricos. Para el encaminamiento de esta revisión sistemática fueron seguidos los criterios de los Artículos de Información Preferido para Revisiones Sistemáticas y Meta-Análisis (Declaración PRISMA) en inglés Preferred Reporting Items for Systematic Reviews and Meta-Analyses (PRISMA Statement). Para búsquedas se optó por las bases de datos CINAHL, LILACS, MEDLINE y EMBASE y se obtuvo una muestra final de 33 artículos en portugués, español e inglés, sin delimitación de tiempo para la búsqueda y con exclusión de los que abordasen pañales infantiles. Las investigaciones pusieron de relieve las características relacionadas con la materia prima y funcionalidad del pañal, así como el uso de técnicas y las lagunas en la normalización de este proceso en las intervenciones de enfermería. Del mismo modo, los estudios han demostrado que las afecciones de la piel y la incontinencia urinaria están relacionadas con la calidad de los productos utilizados y la frecuencia de los cambios.

DESCRIPTORES: Pañales para adultos. Cuidados de Enfermería. Enfermería geriátrica.

\section{INTRODUÇÃO}

O uso de fraldas por idosos hospitalizados pode ser considerado um dos responsáveis pelo surgimento de agravos dermatológicos e exacerbação dos episódios de incontinência urinária. A ausência de padronização quanto ao seu uso e indicação contribui para a presença dos eventos adversos. Assim, estabelecer parâmetros em relação ao tipo e formato ajustado ao paciente, à capacidade e velocidade de absorção, ao período de saturação, aos inibidores de odores, à rotina de limpeza para promoção do conforto são essenciais para uma prática segura ${ }^{1}$.

Os produtos absorventes para a higiene íntima utilizados ainda se encontram na fase de estudos experimentais para estabelecer protocolos de mensuração da perda real de urina e, de acordo com a Sociedade Internacional de Continência (ICS), o teste recomendado é o pad test, que vale-se de critérios que avaliam a frequencia e quantidade da perda urinária ${ }^{2}$.

Entretanto, não é prática cotidiana o uso dessa avaliação individual em cada idoso para adequar o grau de incontinência com o tipo de fralda. Os fabricantes classificam os produtos de acordo com a incontinência; todavia, se desconhece quais os parâmetros utilizados para estabelecer o grau de incontinência. Ou seja, não se sabe se o grau que indica a classificação do fabricante é similar ao grau indicado pela Sociedade Internacional de Continência (ICS).

Dessa forma, identifica-se uma lacuna no conhecimento produzido quanto à abordagem e relação do uso de fraldas geriátricas e os processos da assistência de enfermagem a idosos hospitalizados. Assim, faz-se necessário a busca na literatura científica de estudos que demonstrem o conhecimento sobre as fraldas, consideradas como produtos de higiene continuamente utilizados na prática, mas sem uma determinação de seus parâmetros técnicos, uso associado de artigos absorventes e produtos de proteção cutânea; assim como os produtos e maneiras de utilizá-los. Este estudo tem como objetivo analisar o uso da fralda geriátrica ao que concerne a técnica, os produtos e os eventos adversos e a partir disso formular um protocolo de intervenção para uso de fraldas geriátricas.

\section{MÉTODO}

Trata-se de um estudo de revisão sistemática, conduzido conforme a metodologia Preferred Reporting Items for Systematic Reviews and Meta-Analyses (PRISMA) ${ }^{3}$, que buscou a contextualização das fraldas como produto/estrutura do cuidado de enfermagem e as práticas/técnicas/processos vinculados ao seu uso. Para formulação da pergunta de pesquisa adotou-se a estratégia $\mathrm{PICO}^{4}$ :

- Population (População): adultos e idosos;

- Intervention (Intervenção): parâmetros técnicos do uso de fraldas geriátricas;

- Comparation (Comparação): técnica assistemática do uso de fraldas geriátricas;

- Outcomes (resultados/desfechos): eventos adversos: presença de dermatite por incontinência, úlcera por pressão, surgimento/manutenção da incontinência urinária e interferência na qualidade de vida. 
Para seleção dos estudos foram consultadas as seguintes bases de dados: Cumulative Index to Nursing and Allied Health (CINAHL), Medical Literature Analysis and Retrieval System Online (MEDLINE), Literatura Latino-Americana em Ciências da Saúde (LILACS) e Embase Database (EMBASE) com os descritores propostos pela biblioteca Virtural de Saúde (BVS): 'Fraldas para adultos'/'Adult, diapers'e, 'Idoso'/Aged'. E, quando apropriado com a Terminologia Medical Subject Headings (MeSH): 'Adult, diapers' e 'Aged'. Para busca, os descritores foram associados de duas formas:

1. 'Fraldas para adultos'/'Adult, diapers' com o descritor 'Idoso'/Aged' e

2. o descritor 'Fraldas para adultos'/'Adult, diapers' isolado.

Os artigos foram selecionados de acordo com os critérios de inclusão: Artigos com foco de investigação sobre intervenções de enfermagem a idosos que utilizam fralda na hospitalização; Artigos que descrevam a fralda geriátrica como produto e materiais que a compõem; Artigos que descrevem a fralda geriátrica inserida nas práticas, técnicas e intervenções de enfermagem; Artigos publicados nas línguas portuguesa, inglesa e espanhola. Não há delimitação temporal para seleção dos artigos. Critérios de exclusão: Artigos relacionados a intervenções de enfermagem relacionadas ao uso de fraldas em crianças. Artigos relacionados à fralda infantil como produto.
Para avaliação dos artigos, utilizou-se da proposta PRISMA que contém os itens relevantes para serem identificados na revisão. Para análise do material encontrado, optou-se por categorizar as informações de acordo com os dados sobre a fralda como produto utilizado nas práticas de enfermagem e saúde; materiais que compões as fraldas geriátricas; o modo de utilizar e técnicas comuns ao uso de fraldas em idosos hospitalizados; e quais os eventos ou complicações decorrentes do uso de fraldas.

No total, foram identificados 414 artigos; 288 na base CINAHL, 121 na MEDLINE, 3 na LILACS e 2 na EMBASE. Neste momento, houve leitura do resumo dos artigos para seleção de acordo com os critérios estabelecidos com redução da amostra para 91 artigos na CINAHL, 52 na MEDLINE, 2 na LILACS e 1 na EMBASE (Figura 1).

Após a leitura dos artigos e captação das informações pertinentes, foram criadas categorias de análise dos temas recorrentes: a fralda geriátrica como produto absorvente para eliminações urinária; materiais e funções; e utilização da fralda em idosos ao que se refere ao modo de usar, práticas de enfermagem com a fralda geriátrica, adequabilidade e efeitos do uso, o uso por gênero e outros produtos utilizados concomitantes à fralda. Estes sumarizados nas categorias de análise: Fraldas como produto do cuidado de enfermagem; Técnica e processo no uso de fraldas por

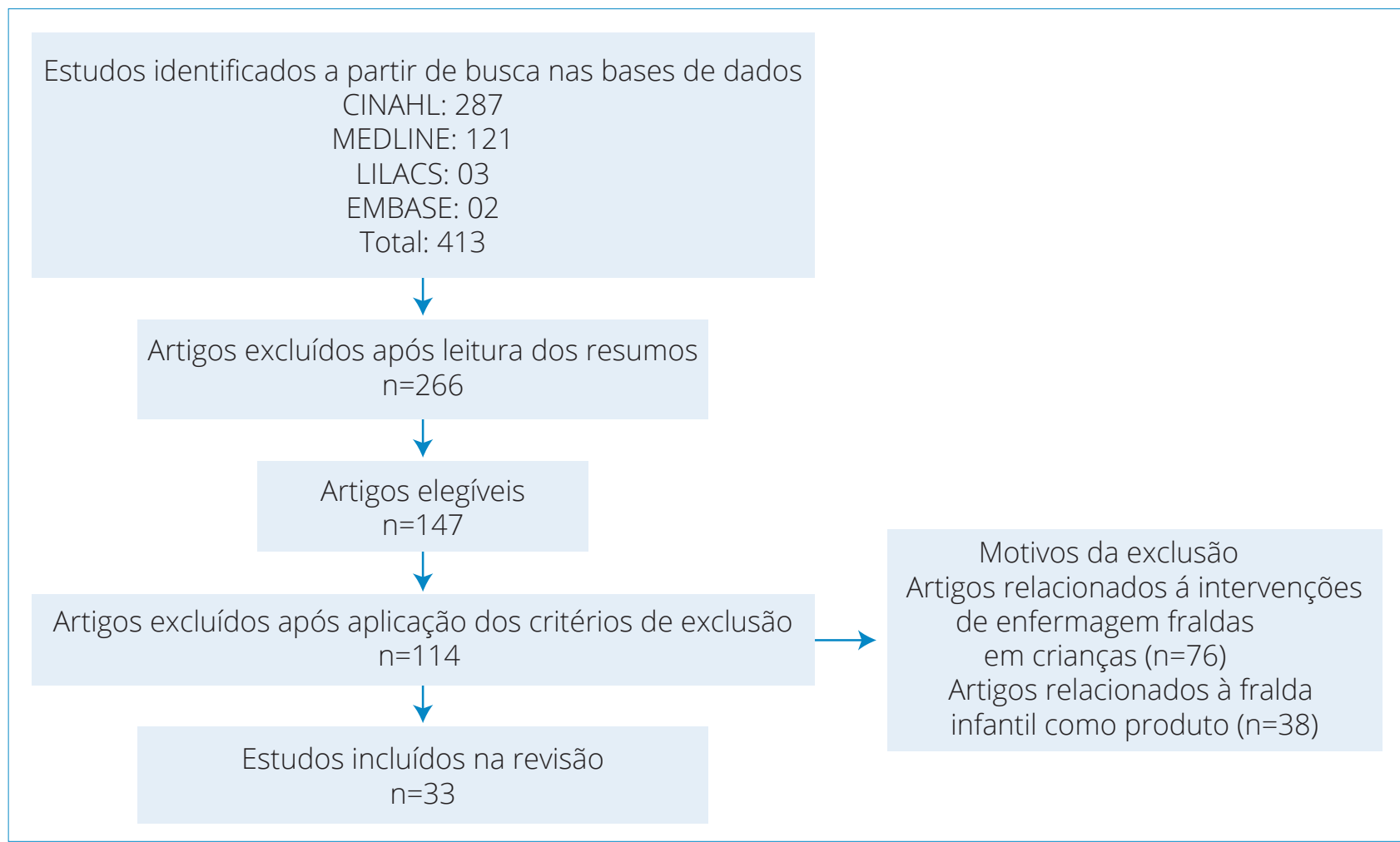

Figura 1. Fluxograma de identificação e seleção dos artigos para revisão sistemática, Niterói, 2015. 
idosos hospitalizados; Eventos adversos associados ao uso de fraldas: Dermatite Associada à Incontinência, Úlcera por pressão, qualidade de vida, dor e desconforto.

\section{RESULTADOS}

Houve uma maioria dos artigos na língua inglesa (91,6\%), com período de publicação de 2004 a 2008 como os mais prevalentes. Identificou-se metade da amostra como estudos de revisão da literatura, e 13,8\% daqueles caracterizados com ensaios clínicos randomizados. A Tabela 1 descreve os artigos de acordo com o tipo de estudo, nível de evidência, autores, ano de publicação, objetivos do estudo e a base de dados encontrada.

Como produto de análise da revisão da literatura, obteve-se a formulação da 'Intervenção padrão'(Quadro 1).

\section{DISCUSSÃO}

\section{Fraldas como produto do cuidado de enfermagem}

De acordo com alguns autores, há entre as categorias dos produtos duas subdivisões: os que são descartáveis (de uso único) e aqueles reutilizáveis (laváveis). Pode-se catalogar conforme o desenho e a adaptação ao formato do corpo e, principalmente, de acordo com o grau de incontinência ${ }^{5}$.

Os produtos absorventes descartáveis ou laváveis, geralmente, compreendem três camadas principais compostas por: um núcleo absorvente prensado entre a base impermeável do produto e a camada superior (permeável) que entra em contato com a pele do indivíduo. $\mathrm{O}$ componente principal desse núcleo absorvente é invariavelmente algum tipo de conjunto de fibras organizadas que contém adicionalmente determinado material superabsorvente, por vezes referido como polímero superabsorvente (PSA) ou absorvente de material gelificante (AMG). Estes, frequentemente, se concentram na região perineal, com variabilidade quanto ao equilíbrio das propriedades químicas, alterações na capacidade e velocidade de absorção desejadas ${ }^{6}$.

Em contrapartida, em relação a outros produtos absorventes, o ideal seria optar pelos absorventes próprios para urina, pois estes são mais eficazes em comparação àqueles usados para período menstrual. Justifica-se essa afirmativa pelo fato de possuírem uma área de superfície que é contrária ao períneo e que assim favorece o escoamento da urina para o núcleo do absorvente e ainda contém polímeros de absorção evitando o extravasamento da urina e diminuindo odores ${ }^{7}$.

No caso de idosos hospitalizados, a escolha de modelos apropriados para as necessidades de incapacidade física e cognitiva auxiliam na minimização de agravos dermatológicos

Quadro 1. Descrição da 'Intervenção padrão: uso de Fraldas' segundo a literatura. Niterói, 2015.

Intervenção padrão: uso de fraldas

1. Separar o material necessário para a troca da fralda:

- fralda geriátrica descartável;

- absorvente urinário (se desejar);

- produto de barreira cutâneo de escolha;

- panos limpos e descartáveis ou algodão hidrofílico;

- luva de procedimento.

2. Retirar a fralda suja evitando o contato das excretas com a região limpa.

3. Limpar a pele perineal com panos úmidos com água limpa em temperatura ambiente sempre em sentido do órgão genital para o ânus. Não reutilizar o pano.

4. Evitar friccionar a pele excessivamente. Para remoção de produtos de barreira, aumentar a frequência da limpeza em detrimento da força em cada limpeza.

5. Evitar o uso de bacias de banho para o cuidado perineal; essas bacias são frequentemente contaminadas. Exceto no caso que a bacia esteja em envelope estéril.

6. Conferir as dobras da pele para fezes e urina residual; caso haja, remover como descrito anteriormente. Atentar para que a pele mantenha-se menos úmida possível.

7. Após a limpeza, utilizar o produto de barreira selecionado (por exemplo, pomada de óxido de zinco).

8. Utilizar a fralda no tamanho anatômico adequado ao idoso, fixando o absorvente de urina na região frontal. Não utilizar mais de uma fralda e verificar se foi fechada adequadamente.

8. Utilizar a fralda no tamanho anatômico adequado ao idoso, fixando o absorvente de urina na região frontal. Não utilizar mais de uma fralda e verificar se foi fechada adequadamente. 9. Educar todos os prestadores de cuidados no método preferido de cuidados da pele.

10. As trocas devem ser realizadas a cada eliminação urinária e/ou intestinal voluntária ou por incontinência. Não exceder o período de duas horas para troca após episódio de eliminação. 
e influência na qualidade de vida ${ }^{5}$. Dessa maneira, o conhecimento das características e parâmetros que determinam o produto ideal tornou-se primordial para a escolha da fralda a ser utilizada, na medida em que favorece à prevenção das injúrias na pele e ocasiona menos desconforto ao paciente.

\section{Técnica e processo no uso de fraldas por idosos hospitalizados}

Tão importante quanto o produto é o modo de utilização, assim como o processo desenvolvido para seu uso, os parâmetros estabelecidos de adequação, validade, benefícios, riscos e a segurança.

$\mathrm{O}$ ambiente perineal pode ser alterado pela frequência e tipo de incontinência, pela condição da pele (inflamação e edema). Os fatores que prejudicam a pele perineal são aqueles como hidratação, $\mathrm{pH}$ da urina e/ou fezes e patógenos já presentes nas fezes ou na microbiota da pele. Há de se destacar ainda que a capacidade cognitiva do idoso de reconhecer a necessidade de ir ao sanitário compreende influência nesse processo ${ }^{8}$.

A utilização de produtos de barreira favorece a proteção cutânea, assim como o aumento da frequência de troca das fraldas diminui os agravos à pele. Porém, não há uma recomendação clara sobre qual seria o número de trocas ou o intervalo adequado ${ }^{9-14}$.

Sobre os produtos como pomadas e emolientes, relatou-se a ligação da Dermatite Associada a Incontinência (DAI) com a necessidade de utilização desses produtos tópicos. Assim, a rotina de cuidado da pele deve ser baseada no aumento da frequência de troca das fraldas e no uso exclusivo de água morna e sabão neutro para limpeza da região genital. A secagem deve ser feita de forma suave, evitando friccionar a região, e, finalmente, deve-se aplicar o protetor cutâneo ou antifúngico tópico em caso de infecções secundárias ${ }^{11}$.

Em sua maioria, os estudos não relatam objetivamente qual seria o tempo ideal para se trocar a fralda ou se há algum aspecto determinante na avaliação do enfermeiro para estabelecer o intervalo de troca ${ }^{14}$. Assim, apesar da lacuna que se apresenta em relação à frequência e intervalo de troca das fraldas, há estudos que destacam que o tempo necessário para se produzir alguma reação eritematosa na pele quando em contato com urina ou fezes é de duas horas; e que o volume de urina (valores maiores que $50 \mathrm{~mL}$ ) é mais decisivo para o aparecimento de dermatites e infecções do que a quantidade de troca da fralda.
Desta forma, tão importante como o produto é o processo de como usar as fraldas no cotidiano do trabalho da enfermagem, visto que esses produtos podem ser utilizados durante períodos prolongados e as medidas adequadas de manejo e cuidados com a pele podem manter a integridade ou diminuir os riscos de lesões independente do período de uso.

\section{Eventos adversos associados ao uso de fraldas: Dermatite Associada à Incontinência e úlcera por pressão}

A dermatite associada à incontinência (DAI) pode ser definida como um acometimento com presença de eritema e edema na superfície da pele, por vezes, acompanhado por bolhas de exsudato seroso, erosão ou infecção cutânea secundária. Por ser reconhecida há pouco tempo como uma forma distinta de danos à pele, as investigações sobre epidemiologia, etiologia, fisiopatologia, prevenção e tratamento ainda caminham na produção desses conhecimentos ${ }^{15}$.

As úlceras por pressão (UP) são lesões cutâneas provocadas por fatores externos, encontram-se como importantes eventos que acometem a pele da região pélvica. Entretanto, a distinção entre UP e DAI é clara à medida que as úlceras por pressão, geralmente, se desenvolvem sobre proeminências ósseas e tendem a assumir a forma de pressão causando, assim, os danos tissulare ${ }^{15,16}$.

A umidade causada pelo uso prolongado de fraldas diminui a resistência da pele, sendo um fator contribuinte para o surgimento das UPP, especialmente para aqueles idosos restritos ao leito e com mobilidade física prejudicada. $\mathrm{O}$ argumento clinicamente relevante para diferenciar DAI de UP é o impacto da prevenção e tratamento. Nota-se que, por vezes, na adoção de um regime de cuidados da pele definido para todos os pacientes, apenas aqueles em risco para DAI acabam recebendo adição de um protetor de pele ao seu regime. E, assim, tem-se a percepção que esse regime também é favorável para UP e, pode preveni-la ${ }^{16}$.

Dentre os estudos realizados sobre DAI, os resultados sugerem que existe associação entre incontinência urinária e danos à pele nas áreas expostas ${ }^{16}$. Portanto, é razoável concluir que os pacientes que são incontinentes estão em maior risco para o aparecimento dessas lesões.

Há concordância na literatura sobre a umidade da pele e a exposição prolongada a qualquer tipo de solução salina, água ou urina atuar como irritante e resultar na dermatite. Esse contato é capaz de provocar danos, habitualmente, 
Tabela 1. Distribuição dos estudos segundo autores e ano de publicação, periódico, tipo de estudo, nível de evidência, base de dados e objetivo do estudo. Niterói, 2015.

\begin{tabular}{|c|c|c|c|c|c|c|}
\hline Código & Artigo & Periódico & $\begin{array}{c}\text { Tipo de } \\
\text { publicação }\end{array}$ & $\begin{array}{l}\text { Nível de } \\
\text { evidência }\end{array}$ & $\begin{array}{c}\text { Base de } \\
\text { dados }\end{array}$ & Objetivos \\
\hline 01 & $\begin{array}{c}\text { Sugama et al. } \\
(2012)^{11}\end{array}$ & $\begin{array}{c}\text { BMC } \\
\text { Geriatrics }\end{array}$ & $\begin{array}{l}\text { Ensaio clínico } \\
\text { randomizado }\end{array}$ & $1 \mathrm{~B}$ & MEDLINE & $\begin{array}{c}\text { Comparar dois produtos } \\
\text { absorventes em idosas que } \\
\text { apresentaram dermatite por } \\
\text { incontinência }\end{array}$ \\
\hline 02 & $\begin{array}{l}\text { Beguin et al. } \\
\qquad(2010)^{6}\end{array}$ & $\begin{array}{c}\text { BMC } \\
\text { Geriatrics }\end{array}$ & $\begin{array}{l}\text { Ensaio clínico } \\
\text { randomizado }\end{array}$ & $1 \mathrm{~B}$ & MEDLINE & $\begin{array}{l}\text { Testar como a forma } \\
\text { modificada do desenho da } \\
\text { fralda afeta parâmetros de } \\
\text { barreira epidérmica }\end{array}$ \\
\hline 03 & $\begin{array}{c}\text { Fader et al. } \\
(2008)^{5}\end{array}$ & $\begin{array}{l}\text { Health } \\
\text { Technology } \\
\text { Assessment }\end{array}$ & $\begin{array}{l}\text { Ensaio clínico } \\
\text { randomizado } \\
\text { multicêntrico }\end{array}$ & $1 \mathrm{~B}$ & MEDLINE & $\begin{array}{l}\text { Comparar o desempenho } \\
\text { e a relação custo-eficácia } \\
\text { dos principais projetos } \\
\text { de produtos absorventes. } \\
\text { Realizar a primeira fase do } \\
\text { desenvolvimento de um } \\
\text { instrumento de qualidade } \\
\text { de vida (QV) para medir o } \\
\text { impacto do uso do produto } \\
\text { absorvente }\end{array}$ \\
\hline 04 & $\begin{array}{l}\text { Drennan et al. } \\
(2012)^{15}\end{array}$ & $\begin{array}{c}\text { BMC } \\
\text { Geriatrics }\end{array}$ & $\begin{array}{l}\text { Revisão } \\
\text { Sistemática da } \\
\text { Literatura }\end{array}$ & $2 \mathrm{~A}$ & MEDLINE & $\begin{array}{c}\text { Identificar intervençôes } \\
\text { conservadoras para } \\
\text { incontinência em pessoas } \\
\text { com demência ou prejuízo } \\
\text { cognitivo que vivem em casa }\end{array}$ \\
\hline 05 & $\begin{array}{l}\text { Fader et al. } \\
(2008)^{16}\end{array}$ & $\begin{array}{l}\text { Health } \\
\text { Technology } \\
\text { Assessment }\end{array}$ & $\begin{array}{l}\text { Revisão } \\
\text { Sistemática da } \\
\text { Literatura }\end{array}$ & $1 \mathrm{~A}$ & MEDLINE & $\begin{array}{c}\text { Para avaliar a eficácia dos } \\
\text { diferentes tipos de produtos } \\
\text { absorventes concebidos } \\
\text { para incontinência } \\
\text { moderada/ pesada }\end{array}$ \\
\hline 06 & $\begin{array}{l}\text { Newman et al. } \\
(2004)^{1}\end{array}$ & $\begin{array}{l}\text { Nursing } \\
\text { Research }\end{array}$ & $\begin{array}{l}\text { Revisão } \\
\text { Narrativa da } \\
\text { Literatura }\end{array}$ & - & MEDLINE & $\begin{array}{l}\text { Analisar produtos e } \\
\text { dispositivos utilizados para } \\
\text { a incontinência e identificar } \\
\text { caminhos para a pesquisa } \\
\text { e desenvolvimento em } \\
\text { tecnologia }\end{array}$ \\
\hline 07 & $\begin{array}{l}\text { Newman } \\
(2004)^{7}\end{array}$ & $\begin{array}{l}\text { Urology } \\
\text { Nursing }\end{array}$ & $\begin{array}{l}\text { Revisão } \\
\text { Narrativa da } \\
\text { Literatura }\end{array}$ & - & MEDLINE & $\begin{array}{l}\text { Descrever os dispositivos de } \\
\text { coleta e produtos utilizados } \\
\text { por idosos incontinentes }\end{array}$ \\
\hline 08 & $\begin{array}{l}\text { Wolf et al. } \\
(2001)^{9}\end{array}$ & $\begin{array}{l}\text { Clinics in } \\
\text { Dernatoloy }\end{array}$ & $\begin{array}{l}\text { Revisão } \\
\text { Narrativa da } \\
\text { Literatura }\end{array}$ & - & MEDLINE & $\begin{array}{c}\text { Descrever a etiologia da } \\
\text { dermatite por fraldas e } \\
\text { implicações na prevenção e } \\
\text { tratamento }\end{array}$ \\
\hline 09 & $\begin{array}{l}\text { Tarbox et al. } \\
(2004)^{17}\end{array}$ & $\begin{array}{l}\text { Journal of } \\
\text { the Applied } \\
\text { Behavior } \\
\text { Analysis }\end{array}$ & $\begin{array}{l}\text { Estudo de } \\
\text { caso }\end{array}$ & 4 & MEDLINE & $\begin{array}{l}\text { Investigar os efeitos } \\
\text { comportamentais } \\
\text { indesejados em decorrência } \\
\text { da fralda }\end{array}$ \\
\hline 10 & $\begin{array}{c}\text { Rogers et al. } \\
(2008)^{18}\end{array}$ & $\begin{array}{l}\text { Journal of } \\
\text { the American } \\
\text { Geriatrics }\end{array}$ & $\begin{array}{l}\text { Ensaio clínico } \\
\text { randomizado }\end{array}$ & $1 \mathrm{~B}$ & MEDLINE & $\begin{array}{l}\text { Avaliar o uso de dispositivos } \\
\text { de coleta de urina e examinar } \\
\text { preditores de avaliação em } \\
\text { centros de saúde }\end{array}$ \\
\hline
\end{tabular}


Tabela 1. Continuação.

\begin{tabular}{|c|c|c|c|c|c|c|}
\hline Código & Artigo & Periódico & $\begin{array}{c}\text { Tipo de } \\
\text { publicação }\end{array}$ & $\begin{array}{l}\text { Nível de } \\
\text { evidência }\end{array}$ & $\begin{array}{c}\text { Base de } \\
\text { dados }\end{array}$ & Objetivos \\
\hline 11 & $\begin{array}{c}\text { Black et al. } \\
(2011)^{12}\end{array}$ & $\begin{array}{l}\text { Journal } \\
\text { Wound } \\
\text { Ostomy } \\
\text { Continence } \\
\text { Nursing }\end{array}$ & $\begin{array}{l}\text { Revisão } \\
\text { Narrativa da } \\
\text { Literatura }\end{array}$ & - & MEDLINE & $\begin{array}{l}\text { Rever o conhecimento atual } \\
\text { de danos à pele associados } \\
\text { à umidade e fornecer } \\
\text { recomendações para a } \\
\text { prevenção e gestão }\end{array}$ \\
\hline 12 & $\begin{array}{l}\text { Foureur } \\
\text { et al. } \\
(2006)^{10}\end{array}$ & $\begin{array}{l}\text { British } \\
\text { Journal of } \\
\text { Dermatology }\end{array}$ & $\begin{array}{c}\text { Estudo } \\
\text { observacional }\end{array}$ & - & MEDLINE & $\begin{array}{l}\text { Determinar as causas da } \\
\text { dermatite por incontinência } \\
\text { em idosos e para avaliar a } \\
\text { eficácia dos tratamentos } \\
\text { antifúngicos nesta indicação }\end{array}$ \\
\hline 13 & $\begin{array}{l}\text { Roe et al. } \\
(2011)^{19}\end{array}$ & $\begin{array}{l}\text { Journal of } \\
\text { Advanced } \\
\text { Nursing }\end{array}$ & $\begin{array}{c}\text { Revisão } \\
\text { sistemática } \\
\text { da literatura }\end{array}$ & $1 \mathrm{~A}$ & MEDLINE & $\begin{array}{c}\text { Identificar estudos descritivos } \\
\text { com a incontinência como o } \\
\text { principal foco em idosos } \\
\text { em lare }\end{array}$ \\
\hline 14 & $\begin{array}{l}\text { Aquino et al. } \\
\qquad(2012)^{20}\end{array}$ & $\begin{array}{c}\text { Revista } \\
\text { Eletrônica de } \\
\text { Enfermagem }\end{array}$ & $\begin{array}{l}\text { Revisão } \\
\text { integrativa da } \\
\text { literatura }\end{array}$ & - & LILACS & $\begin{array}{c}\text { Identificar na literatura as } \\
\text { melhores intervenções para } \\
\text { o tratamento e prevenção } \\
\text { da integridade da pele } \\
\text { prejudicada relacionada ao } \\
\text { uso de fraldas evidenciada } \\
\text { por dermatite }\end{array}$ \\
\hline 15 & $\begin{array}{l}\text { Duarte et al. } \\
\qquad(2007)^{21}\end{array}$ & $\begin{array}{l}\text { Anais } \\
\text { Brasileiros de } \\
\text { Dermatologia }\end{array}$ & $\begin{array}{l}\text { Estudo } \\
\text { experimental }\end{array}$ & - & LILACS & $\begin{array}{l}\text { Verificar a frequência da } \\
\text { dermatite de contato em } \\
\text { idosos, num grupo de } \\
\text { pacientes com hipótese } \\
\text { diagnóstica de dermatite } \\
\text { de contato; demonstrar os } \\
\text { principais sensibilizantes nesse } \\
\text { grupo; comparar os dados } \\
\text { obtidos nos testes de contato, } \\
\text { com um grupo de adultos } \\
\text { atendidos no mesmo período }\end{array}$ \\
\hline 16 & $\begin{array}{c}\text { Miyamaru } \\
\text { et al. }(2006)^{22}\end{array}$ & $\begin{array}{l}\text { Revista } \\
\text { Instituto } \\
\text { Adolfo Luiz }\end{array}$ & $\begin{array}{c}\text { Estudo } \\
\text { observacional }\end{array}$ & - & EMBASE & $\begin{array}{l}\text { Comparar os métodos in } \\
\text { vivo e in vitro na avaliação da } \\
\text { toxicidade dérmica e estimar } \\
\text { a qualidade microbiológica } \\
\text { de } 60 \text { amostras de produtos } \\
\text { de higiene descartáveis } \\
\text { comercializados na cidade } \\
\text { de São Paulo }\end{array}$ \\
\hline 17 & $\begin{array}{l}\text { Bliss e } \\
\text { Hurlow } \\
(2011)^{23}\end{array}$ & $\begin{array}{l}\text { Geriactrics } \\
\text { Nursing }\end{array}$ & $\begin{array}{l}\text { Revisão } \\
\text { Narrativa da } \\
\text { Literatura }\end{array}$ & - & CINAHL & $\begin{array}{l}\text { Identificar e descrever os } \\
\text { produtos e intervenções } \\
\text { utilizados no manejo da pela } \\
\text { seca em idosos }\end{array}$ \\
\hline 18 & $\begin{array}{c}\text { Cottenden } \\
(1988)^{24}\end{array}$ & $\begin{array}{l}\text { Journal of } \\
\text { Medical and } \\
\text { Biological } \\
\text { Engineering }\end{array}$ & $\begin{array}{l}\text { Revisão } \\
\text { Narrativa da } \\
\text { Literatura }\end{array}$ & - & CINAHL & $\begin{array}{l}\text { Descrever os requisitos } \\
\text { funcionais de absorventes } \\
\text { de incontinência urinária e } \\
\text { analisar os resultados de } \\
\text { vários estudos que procuram } \\
\text { relacionar o desempenho } \\
\text { clínico desses produtos }\end{array}$ \\
\hline
\end{tabular}


Tabela 1. Continuação.

\begin{tabular}{|c|c|c|c|c|c|c|}
\hline Código & Artigo & Periódico & $\begin{array}{c}\text { Tipo de } \\
\text { publicação }\end{array}$ & $\begin{array}{c}\text { Nível de } \\
\text { evidência }\end{array}$ & $\begin{array}{c}\text { Base de } \\
\text { dados }\end{array}$ & Objetivos \\
\hline 19 & $\begin{array}{l}\text { Pfisterer et al. } \\
(2007)^{25}\end{array}$ & $\begin{array}{l}\text { Journal } \\
\text { American } \\
\text { Geriatrics } \\
\text { Society }\end{array}$ & $\begin{array}{c}\text { Estudo } \\
\text { observacional }\end{array}$ & - & CINAHL & $\begin{array}{l}\text { Analisar as preferências } \\
\text { para diferentes tipos } \\
\text { de tratamento de } \\
\text { incontinência urinária em } \\
\text { pacientes geriátricos }\end{array}$ \\
\hline 20 & $\begin{array}{l}\text { Engerg et al. } \\
(2004)^{26}\end{array}$ & $\begin{array}{l}\text { Nursing } \\
\text { Research }\end{array}$ & $\begin{array}{l}\text { Revisão } \\
\text { Narrativa da } \\
\text { Literatura }\end{array}$ & - & CINAHL & $\begin{array}{c}\text { Resumir o conhecimento } \\
\text { atual sobre incontinência } \\
\text { urinária em idosos } \\
\text { mais velhos }\end{array}$ \\
\hline 21 & $\begin{array}{l}\text { Junkin e } \\
\text { Selekof } \\
(2007)^{14}\end{array}$ & $\begin{array}{l}\text { Journal Wound } \\
\text { Ostomy } \\
\text { Continence } \\
\text { Nursing }\end{array}$ & Estudo piloto & - & CINAHL & $\begin{array}{c}\text { Identificar a prevalência } \\
\text { de incontinência e lesões } \\
\text { na pele em áreas expostas } \\
\text { a incontinência entre } \\
\text { pacientes internados em } \\
\text { dois hospitais }\end{array}$ \\
\hline 22 & $\begin{array}{l}\text { Figueiredo } \\
\text { et al. }(2012)^{27}\end{array}$ & $\begin{array}{l}\text { International } \\
\text { Urogynecolo-gy } \\
\text { Journal }\end{array}$ & $\begin{array}{c}\text { Estudo } \\
\text { observacional }\end{array}$ & - & CINAHL & $\begin{array}{l}\text { Avaliar a perda de urina } \\
\text { em mulheres que usam } \\
\text { absorventes urinários }\end{array}$ \\
\hline 23 & $\begin{array}{l}\text { Zurcher et al. } \\
\qquad(2011)^{28}\end{array}$ & $\begin{array}{l}\text { Nursing } \\
\text { Research and } \\
\text { Practice }\end{array}$ & $\begin{array}{c}\text { Estudo } \\
\text { transversal }\end{array}$ & - & CINAHL & $\begin{array}{l}\text { Avaliar práticas de } \\
\text { enfermagem sobre } \\
\text { pacientes idosos } \\
\text { hospitalizados com } \\
\text { incontinência urinária, } \\
\text { a fim de determinar } \\
\text { a necessidade de } \\
\text { melhoria da qualidade } \\
\text { ou redirecionamento do } \\
\text { atendimento }\end{array}$ \\
\hline 24 & $\begin{array}{c}\text { Weiss } \\
(1998)^{29}\end{array}$ & $\begin{array}{l}\text { American } \\
\text { Family } \\
\text { Physician }\end{array}$ & $\begin{array}{l}\text { Revisão } \\
\text { Narrativa da } \\
\text { Literatura }\end{array}$ & - & CINAHL & $\begin{array}{l}\text { Descreve a avaliação da } \\
\text { incontinência urinária em } \\
\text { pacientes geriátricos, com } \\
\text { foco em uma abordagem } \\
\text { de diagnóstico para uso em } \\
\text { cuidados primários }\end{array}$ \\
\hline 25 & $\begin{array}{l}\text { Schneider } \\
\text { et al. }(2001)^{30}\end{array}$ & $\begin{array}{l}\text { Journal of } \\
\text { the American } \\
\text { Medical } \\
\text { Association }\end{array}$ & $\begin{array}{l}\text { Estudo de } \\
\text { caso }\end{array}$ & - & CINAHL & $\begin{array}{l}\text { Relatar os sintomas, } \\
\text { evolução e tratamento de } \\
\text { uma idosa de } 71 \text { anos com } \\
\text { incontinência urinária }\end{array}$ \\
\hline 26 & $\begin{array}{l}\text { Bale et al. } \\
(2004)^{31}\end{array}$ & $\begin{array}{c}\text { Journal Tissue } \\
\text { Viability }\end{array}$ & $\begin{array}{c}\text { Estudo } \\
\text { experimental }\end{array}$ & - & CINAHL & $\begin{array}{l}\text { Analisar um novo protocolo } \\
\text { de cuidados da pele em } \\
\text { lares de idosos e seus } \\
\text { efeitos sobre a condição } \\
\text { da pele dos pacientes, } \\
\text { e custos associados }\end{array}$ \\
\hline 27 & $\begin{array}{l}\text { Nix e } \\
\text { Ermer- } \\
\text { Seltum } \\
(2004)^{32}\end{array}$ & $\begin{array}{l}\text { Ostomy Wound } \\
\text { Manage }\end{array}$ & $\begin{array}{l}\text { Revisão } \\
\text { Narrativa da } \\
\text { Literatura }\end{array}$ & - & CINAHL & $\begin{array}{l}\text { Determinar a extensão } \\
\text { na qual os protocolos de } \\
\text { cuidados da pele perineal } \\
\text { são consistentes e estimar } \\
\text { o nível de cumprimento } \\
\text { relacionado com o uso de } \\
\text { protetores da pele perineal }\end{array}$ \\
\hline
\end{tabular}


Tabela 1. Continuação.

\begin{tabular}{|c|c|c|c|c|c|c|}
\hline Código & Artigo & Periódico & $\begin{array}{c}\text { Tipo de } \\
\text { publicação }\end{array}$ & $\begin{array}{c}\text { Nível de } \\
\text { evidência }\end{array}$ & $\begin{array}{c}\text { Base de } \\
\text { dados }\end{array}$ & Objetivos \\
\hline 28 & $\begin{array}{l}\text { Brunner et al. } \\
\qquad(2012)^{33}\end{array}$ & Urology Nursing & $\begin{array}{l}\text { Revisão } \\
\text { Narrativa da } \\
\text { Literatura }\end{array}$ & - & CINAHL & $\begin{array}{l}\text { Comparar dois produtos } \\
\text { de cuidados da pele para } \\
\text { determinar a incontinência } \\
\text { e verificar o mais eficaz } \\
\text { para a prevenção de pele }\end{array}$ \\
\hline 29 & $\begin{array}{l}\text { Eva et al. } \\
(2012)^{34}\end{array}$ & $\begin{array}{l}\text { Gastroenterology } \\
\text { Research an } \\
\text { Practice }\end{array}$ & $\begin{array}{l}\text { Revisão } \\
\text { Narrativa da } \\
\text { Literatura }\end{array}$ & - & CINAHL & $\begin{array}{l}\text { Avaliar a prevalência } \\
\text { de incontinência } \\
\text { fecal, fatores de risco } \\
\text { modificáveis e o efeito } \\
\text { sobre a qualidade de vida }\end{array}$ \\
\hline 30 & $\begin{array}{l}\text { Hashizum } \\
(2004)^{35}\end{array}$ & $\begin{array}{l}\text { Dermatology } \\
\text { nursing }\end{array}$ & $\begin{array}{l}\text { Revisão } \\
\text { Narrativa da } \\
\text { Literatura }\end{array}$ & - & CINAHL & $\begin{array}{l}\text { Descrever o processo de } \\
\text { envelhecimento da pele e } \\
\text { as formas de tratamento } \\
\text { e prevenção de danos }\end{array}$ \\
\hline 31 & $\begin{array}{l}\text { McClish } \\
\text { et al. } \\
(1999)^{36}\end{array}$ & $\begin{array}{l}\text { Journal Wound } \\
\text { Ostomy } \\
\text { Continence } \\
\text { Nursing }\end{array}$ & $\begin{array}{c}\text { Estudo } \\
\text { experimental }\end{array}$ & - & CINAHL & $\begin{array}{c}\text { Examinar o uso e } \\
\text { custo de produtos de } \\
\text { incontinência e relação } \\
\text { com idade, tempo } \\
\text { de incontinência, a } \\
\text { frequência diurna, } \\
\text { índices de gravidade, o } \\
\text { diagnóstico urodinâmico e } \\
\text { qualidade de vida }\end{array}$ \\
\hline 32 & $\begin{array}{l}\text { Thornburn } \\
\text { et al. } \\
(1997)^{37}\end{array}$ & $\begin{array}{l}\text { Journal Wound } \\
\text { Ostomy } \\
\text { Continence } \\
\text { Nursing }\end{array}$ & $\begin{array}{c}\text { Estudo } \\
\text { experimental }\end{array}$ & - & CINAHL & $\begin{array}{l}\text { Examinar a relação entre } \\
\text { as propriedades da fralda } \\
\text { e a sensação de conforto }\end{array}$ \\
\hline 33 & $\begin{array}{c}\text { Gray et al. } \\
(2012)^{13}\end{array}$ & $\begin{array}{l}\text { Journal Wound } \\
\text { Ostomy } \\
\text { Continence } \\
\text { Nursing }\end{array}$ & $\begin{array}{l}\text { Revisão } \\
\text { Narrativa da } \\
\text { Literatura }\end{array}$ & - & CINAHL & $\begin{array}{l}\text { Sintetizar e atualizar } \\
\text { o conhecimento das } \\
\text { recomendações de } \\
\text { melhores práticas para } \\
\text { incontinência associada à } \\
\text { dermatite }\end{array}$ \\
\hline
\end{tabular}

CINAHL: Cumulative Index to Nursing and Allied Health; MEDLINE: Medical Literature Analysis and Retrieval System Online; LILACS: Literatura Latino-Americana em Ciências da Saúde; EMBASE: Embase Database.

pela exposição repetida, e pode ter o agravante se contato com material fecal, que diminui as funções de barreira e proteção da pele ${ }^{14}$.

Portanto, três fatores essenciais podem levar ao aparecimento da DAI: a tolerância do tecido cutâneo, o ambiente perineal e modo de limpeza e higiene dessa região. Além disso, fatores como idade, estado de saúde, estado nutricional, exposição da pele ao cisalhamento ou friç̧ão e temperatura corporal também podem interferir ${ }^{14}$.

Inflamação e edema são eventos que comumente caracterizam a DAI. Outros fatores predisponentes podem estar relacionados com o excesso de hidratação, o pH da urina ou fezes, a presença de patógenos oportunistas da microbiota local e, sobretudo, na frequência de troca das fraldas e limpeza adequada dessa região com uso de produtos protetores cutâneos ${ }^{14}$, daí a importância do estudo do processo/técnica ideal para uso das fraldas.

O tempo necessário para o desenvolvimento da DAI foi de aproximadamente de 6 a $42 \operatorname{dias}^{14}$ (mediana de 13 dias). $\mathrm{O}$ tempo é fator importante visto que indivíduos acamados, em processos crônicos de hospitalização e funcionalmente dependentes são aqueles com maior propensão a desenvolver DAI.

A avaliação da DAI é basicamente descritiva e inclui aspectos como cor da pele, integridade, tamanho da lesão, sintomas de dor, ruptura da pele, hiperemia e flogose. Do mesmo modo, são avaliados quais os agentes irritantes, as condições da pele, aspectos nutricionais e processos infecciosos concomitantes ${ }^{14,15}$. 
$\mathrm{O}$ uso adequado de produtos de barreira aliado a frequência regular na troca de fraldas podem garantir a ausência da DAI para aqueles que ainda não apresentam, mas possuem fatores de risco; e provocar melhora naqueles com afecção já instalada; assim como o manejo durante a limpeza e higiene da região perineal, que favorecem a retirada do material irritante com a preocupação de eliminar qualquer sujidade residual ${ }^{14-16}$.

Logo, torna-se importante a história clínica do paciente para determinar a etiologia da lesão da pele perineal. Isso inclui a avaliação regular da pele, avaliação do risco de úlceras de pressão e identificação de incontinência fecal ou urinária, bem como outras fontes de umidade que podem afetar o risco de danos associados de umidade da pele, como a transpiração excessiva ou uma ferida com alto volume exsudado. De certo modo, as eliminações ocorrem de forma ininterrupta, mas a adesão de cuidados sistemáticos da pele podem definir considerável melhora na integridade cutânea ${ }^{16}$, por isso a importância de se estabelecer parâmetros do processo/técnica de uso das fraldas.

\section{CONCLUSÃO}

A recomendação de um padrão para o uso de fraldas geriátricas deteve a finalidade de estabelecer um modo sistemático e uniforme as intervenções de enfermagem. Os estudos demonstraram que os acometimentos cutâneos e de incontinência urinária possuem relação com a qualidade dos produtos utilizados e com a frequência das trocas.

Para tanto, a formulação de um protocolo de pesquisa para testar a intervenção uso de fraldas geriátricas, estabelece um modo sistemático e uniforme para as intervenções de enfermagem com intuito de diminuir a probabilidade de aparecimento de injúrias de pele e mucosa, bem como aparecimento e exacerbação da incontinência urinária e a percepção de interferência na vida diária.

\section{REFERÊNCIAS}

1. Newman DK, Fader M, Bliss DZ. Managing Incontinence Using Technology, Devices, and Products: Directions for Research. Nurs Res. 2004;53(6 Suppl):S42-8.

2. Pereira VS, Santos JYC, Correia GN, Driusso P. Tradução e validação para a língua portuguesa de um questionário para avaliação da gravidade da incontinência urinária. Rev Bras Ginecol Obstet. 2011;33(4):182-7.

3. Moher D, Liberati A, Tetzlaff J, Altman DG; PRISMA Group Preferred reporting items for systematic reviews and meta-analyses: the PRISMA statement. Ann Intern Med. 2009;151(4):264-9.

4. Greenhalgh T. Como ler artigos científicos: fundamentos da medicina baseada em evidências.2. ed. Porto Alegre: Artmed; 2005.

5. Fader M, Cottenden A, Getliffe K, Gage H, Clarke-O'Neill S, Jamieson $\mathrm{K}$, et al. Absorbent products for urinary/faecal incontinence: a comparative evaluation of key product designs. Health Technol Assess. 2008;12(29):iii-iv.

6. Beguin AM, Malaquin-Pavan E, Guihaire C, Hallet-Lezy AM, Souchon S, Homann V, et al. Improving diaper design to address incontinence associated dermatitis. BMC Geriatr. 2010;10:86.

7. Newman DK. Incontinence products and devices for the elderly. Urol Nurs. 2004;24(4):316-33.

8. Aquino PS, Melo RP, Lopes MVO, Pinheiro AKB. Análise do conceito de tecnologia na enfermagem segundo o método evolucionário. Acta Paul Enferm. 2010;23(5):690-6.

9. Wolf R, Wolf D, Tuzun B, Tuzun Y. Diaper Dermatitis. Clin Dermatol. 2001;18(6):657-60.
10. Foureur NB, Vanzo B, Meaume VS, Senet P. Prospective aetiological study of diaper dermatitis in the elderly. $\mathrm{Br}$ J Dermatol.2006;155(5):941-6.

11. Sugama J, Sanada H, Shigeta Y, Nakagami G, Konya C. Efficacy of an improved absorbent pad on incontinence-associated dermatitis in older women: cluster randomized controlled trial. BMC Geriatr. 2012;12:22.

12. Black JM, Gray M, Bliss DZ, Kennedy-Evans KL, Logan S, Baharestani MM, et al. MASD Part 2: Incontinence-Associated Dermatitis and Intertriginous Dermatitis A Consensus. J Wound Ostomy Continence Nurs. 2011:38(4):359-70.

13. Gray M, Beeckman D, Bliss DZ, Fader M, Logan S, Junkin J, et al. Incontinence-Associated Dermatitis: A Comprehensive Review and Update.J Wound Ostomy Continence Nurs. 2012;39(1):61-74.

14. Junkin J, Selekof JL. Prevalence of Incontinence and Associated Skin Injury in the Acute Care Inpatient. J Wound Ostomy Continence Nurs. 2007;34(3):260-9.

15. Drennan VM, Greenwood N, Cole L, Fader M, Grant R, Rait G, et al. Conservative interventions for incontinence in people with dementia or cognitive impairment, living at home: a systematic review. BMC Geriatr. 2012;12:77.

16. Fader M, Cottenden A, Getliffe K. Absorbent products for moderate-heavy urinary and/or faecal incontinence in women and men. Health Technol Assess. 2008;(4):CD007408.

17. Tarbox RS, Williams LW, Friman PC. Extended diaper wearing: effects on continence in and out of the diaper. J Appl Behav Anal. 2004;37(1):97-100.

18. Rogers MA, Mody L, Kaufman SR, Fries BE, Mcmahon LF, Saint S. Use of urinary collection devices in skilled nursing facilities in five states. J Am Geriatr Soc. 200856(5):854-61. 
19. Roe B, Flanagan L, Jack B, Barrett J, Chung A, Shaw C, et al. Systematic review of the management of incontinence and promotion of continence in older people in care homes: descriptive studies with urinary incontinence as primary focus. J Adv Nurs. 2011;67(2):228-50.

20. Aquino AL, Chianca TCM,Brito RCS. Integridade da pele prejudicada, evidenciada por dermatite da área das fraldas: revisão integrativa. Rev Eletr Enf. [Internet]. 2012;14(2):414-24.

21. Duarte I, Kobata C, Lazzarini R. Dermatite de contato em idosos. An Bras Dermatol. 2007;82(2):135-40.

22. Miyamaru LL, Bárbara MCS, Cruz AS, Ikeda TI Sakuma H, Zenebom O. Estudo comparativo entre os métodos in vivo e in vitro na análise toxicológica de produtos de higiene descartáveis e sua avaliação microbiológica. Rev Inst Adolfo Lutz. 2006;65(2):118-22.

23. Bliss DZ, Hurlow J. Dry Skin in Older Adults. GeriatrNurs. 2011;32(4):257-62.

24. Cottenden AM. Incontinence pads: clinical performance, design and technical properties. J Biomed Eng. 1998;10(6):506-14.

25. Pfisterer MH, JohnsonTM, Jenetzky E, Hauer K, Oster P. Geriatric Patients' Preferences for Treatment of Urinary Incontinence: A Study of Hospitalized, Cognitively Competent Adults Aged 80 and Older. J Am Geriatr Soc. 2007;55(12):2016-22.

26. Engerg S, Kincade J, Thompson D. Future Directions for Incontinence Research With Frail Elders. Nurs Res. 2004;53(6):22-9.

27. Figueiredo EM, Gontijo R, Vaz CT, Baracho E, Fonseca AM, Monteiro MV, et al. The results of a 24-h pad test in Brazilian women. Int Urogynecol J. 2012;23(6):785-9.
28. Zurcher S, Saxer S, Scwendimann R. Urinary Incontinence in Hospitalised Elderly Patients: Do Nurses Recognise and Manage the Problem? Nurs Res Pract. 2011;2011:671302.

29. Weiss BD. Diagnostic Evaluation of Urinary Incontinence in Geriatric Patients. Am Fam Physician. 1998;57(11):2675-84.

30. Schneider D, Glew CM, Dasgupta I, Hofmann MT. Urinary Incontinence in an Elderly Woman. Hosp Physician. 2001;21(22):52-61,70.

31. Bale $S$, Tebble $N$, Jones $V$, Price $P$. The benefits of implementing a new skin care protocol in nursing homes. J Tissue Viability. 2004;14(2):44-50.

32. Nix D, Ermer-Seltum J. A review of perineal skin care protocols and skin barrier product use. Ostomy Wound Manage. 2004;50(12):59-67.

33. Brunner M, Droegemuller C, Rivers S, Deuser WE. Prevention of Incontinence-related Skin Breakdown for Acute and Critical Care Patients. Urol Nurs. 2012;3(4):214-9.

34. Eva HA, Coyne T, Hawes SK, Merikhi L, Naples SP, Kanagarajan $N$, et al. Fecal Incontinence: Prevalence, Severity, and Quality of Life Data from an Outpatient Gastroenterology Practice. Gastroenterol Res Pract. 2012;2012:947694.

35. HashizumeH.SkinAgingandDrySkin.JDermatol.2004;31(8):603-9.

36. Mcclish DK, Wyman JF, Sale PG, Camp J, Earle B. Use and costs of incontinence pads in female study volunteers. Continence Program for Women Research Group. J Wound Ostomy Continence Nurs. 1999;26(4):207-8,210-3.

37. Thornburn P, Fader M, Dean G, Brooks R, Cottenden A. Improving the performance of small incontinence pads: a study of "wet comfort". J Wound Ostomy Continence Nurs. 1997;24(4):219-25. 